\title{
Existence of multimodal standing gravity waves
}

\author{
Gérard Iooss $^{\dagger}$, Pavel Plotnikov \\ $\dagger$ IUF, INLN UMR 6618 CNRS - UNSA, 1361 rte des Lucioles, 06560 Valbonne, France \\ gerard.iooss@inln.cnrs.fr \\ $\ddagger$ Russian academy of Sciences, Lavryentyev pr. 15, Novosibirsk 630090, Russia. \\ plotnikov@hydro.nsc.ru
}

\begin{abstract}
We consider two-dimensional standing gravity waves on the surface of an infinitely deep perfect fluid, the flow being potential. It is known that the linearized problem is completely resonant. Following the method described in [4], we prove the existence of an infinity of multimodal standing gravity waves, corresponding to any choice of asymptotic expansion in powers of the amplitude $\varepsilon$, indicated in [2] and [3]. Each one of these solutions exist for a set of values of $\varepsilon$ being dense in 0 .
\end{abstract}

Key words: nonlinear water waves, standing gravity waves, bifurcation theory, small divisors, complete resonance.

AMS classification: 35B32, 35B34, 76B15, 76B07

\section{Introduction}

This paper follows the paper [3], considering the problem of existence of two-dimensional standing gravity waves on an infinitely deep perfect fluid layer (called "clapotis" in french), periodic in time and in the horizontal coordinate, and symmetric with respect to the vertical axis. In [4], Iooss, Plotnikov and Toland proved the existence of unimodal standing waves (only one dominant mode at the main order $\varepsilon$ ), for a set of amplitudes $\varepsilon$ which is dense at 0 . The complete resonance of the linearized problem allows to think about the existence of multimodal standing waves, which means that at order $\varepsilon$ it might be possible to have a suitable combination of several modes (necessarily solutions of the linearized problem). The paper [3] uses the present formulation of the problem, and gives in particular another complete proof of the possibility to find a large family of approximate solutions for our problem, in the form of asymptotic expansions in powers of the amplitude $\varepsilon$, (same result as in [2]). The present paper adapts the lines of [4] , used for proving the existence of unimodal standing waves, and shows the existence of (nearly) all multimodal solutions which possess the asymptotic expansions found in [3], for a set of amplitudes dense at 0 (see the precise statement in Theorem 1 below).

In the present formulation, there is one dimensionless parameter $1+\mu=g T^{2} / 2 \pi \lambda$ where $g$ is the acceleration of gravity, $T$ is the time period, $\lambda$ is the horizontal wave length, $\mu$ being close to 0 . We indeed look for non trivial doubly $2 \pi$ - periodic solutions of the following second order nonlocal PDE, as deduced from the formulation introduced by Dyachenko et al [1]:

$$
\partial_{t}\left(L_{w^{\prime}} \dot{w}\right)-(1+\mu) \mathcal{H} w^{\prime}+\mathcal{H} \partial_{x}\left\{\frac{1}{D} \mathcal{H}\left(\left(L_{w^{\prime}} \dot{w}\right) \mathcal{H} L_{w^{\prime}} \dot{w}\right)+\left(\mathcal{H} L_{w^{\prime}} \dot{w}\right) \mathcal{H}\left(\frac{L_{w^{\prime}} \dot{w}}{D}\right)\right\}=0,
$$

where $w$ is an unknown function of $(x, t) \in \mathbb{R}^{2}$, the free surface of the waves being given in 
the physical plane by

$$
(\xi, \eta)=(x+\mathcal{H} w(x, t),-w(x, t)), \quad(x, t) \in \mathbb{R}^{2} .
$$

In (1) the operator $\mathcal{H}$ denotes the periodic Hilbert transform with respect to $x: \mathcal{H}\left(e^{i n x}\right)=$ $i \operatorname{sgn}(n) e^{i n x}$, and we denote by a dot or $\partial_{t}$ the time $t$ partial derivative and by a prime or $\partial_{x}$ the space $x$ partial derivative. The function $D$ and the linear operator $L_{w^{\prime}}$ are defined as follows, for $w$ doubly periodic and smooth enough and any $f \in L_{\natural}^{2}=L^{2}(\mathbb{R} / 2 \pi \mathbb{Z})$

$$
\begin{aligned}
L_{w^{\prime}} f & =\left(1+\mathcal{H} w^{\prime}\right) f-w^{\prime} \mathcal{H} f \\
D & =\left(1+\mathcal{H} w^{\prime}\right)^{2}+w^{\prime 2} .
\end{aligned}
$$

For eliminating solutions deduced by shifting time and $x$ origins, we look for solutions of (1) which are even in $x$ and in $t$. Moreover, we restrict our study to solutions $w$ with 0 average, since adding a constant to $w$ gives a solution of the problem corresponding to the same physical solution. Equation (1) may be written as

$$
\mathcal{F}(w, \mu)=0,
$$

where $\mathcal{F}$ is a analytic mapping $H_{\text {吅 }}^{m, e e} \times \mathbb{R} \rightarrow H_{\text {吅 }}^{m-2, e e}, m \geq 3$, where $H_{\text {如 }}^{m, e e}$ is by definition the subspace of functions even in $x$ and in $t$ of the Sobolev space $H_{\text {的 }}^{m}=H^{m}\left\{(\mathbb{R} / 2 \pi \mathbb{Z})^{2}\right\}$. Defining the nonlinear terms $\mathcal{N}_{\geq 2}$, we can write

$$
\mathcal{F}(w, \mu)=\mathcal{L}_{0} w-\mu \mathcal{H} w^{\prime}+\mathcal{N}_{\geq 2}(w)
$$

where

$$
\mathcal{L}_{0} w=\ddot{w}-\mathcal{H} w^{\prime} .
$$

The complete resonance in our problem means that the kernel of $\mathcal{L}_{0}$ is infinite dimensional, here spanned by

$$
\left\{\cos q^{2} x \cos q t ; \quad q \in \mathbb{N}\right\},
$$

which leads to an infinite dimensional bifurcation equation. We gave in [3] an infinite set of asymptotic expansions (where $I$ is any finite subset of $\mathbb{N}$ )

$$
\begin{aligned}
w_{\varepsilon}^{(N)} & =\sum_{1 \leq p \leq N} \varepsilon^{p} w^{(p)}, \quad \mu=\frac{\varepsilon^{2}}{4} \\
w^{(1)} & =\sum_{q \in I} \frac{\varepsilon_{q}}{q^{2}} \cos q^{2} x \cos q t, \quad \varepsilon_{q}= \pm 1
\end{aligned}
$$

which are approximate solutions of (2):

$$
\mathcal{F}\left(w_{\varepsilon}^{(N)}, \frac{\varepsilon^{2}}{4}\right)=O\left(\varepsilon^{N+1}\right) \in H_{\text {如 }}^{m, e e}, \text { for any } N \text { and } m .
$$

Let us state our main result:

Theorem 1 For any finite subset I of $\mathbb{N}$, satisfying the following hypothesis

$H(I)$ : For any fixed $p \in I$, the following inequality holds

$$
\sum_{q \in I, q>p} \frac{p^{2}\left(q^{2}-p^{2}\right)}{q^{4}} \neq \frac{1}{2}
$$

there exists a measurable set $\mathcal{E}_{I} \subset\left[0, \varepsilon_{0}\right]$ which is dense at 0 (0 is a Lebesgue point) such that, for any $\varepsilon \in \mathcal{E}_{I}$, there exists a solution $w \in H_{\text {娼 }}^{17, e e}$ with 0 average, of equation (1), with $\mu=\varepsilon^{2} / 4$. The function $\varepsilon \mapsto w$ satisfies $w=w_{\varepsilon}^{(N)}+O\left(\varepsilon^{N}\right)$, for $N \geq 4$, where $w_{\varepsilon}^{(N)}$ is given by (3). 
Remark: This theorem completes the main theorem of [4] where only the case $I=$ $\{1\}$ was considered. We may notice that when $I$ contains one, or two, or three elements, hypothesis $\mathrm{H}(\mathrm{I})$ is satisfied for any choice of numbers.

The method of proof rests on the use of the Nash-Moser implicit function theorem to seek solutions as perturbations of the approximate solutions $w_{\varepsilon}^{(N)}$. As shown in [4], the existence question can be reduced to one of estimating the inverses of linearized operators at non-zero points. Then, there are two difficulties for this inversion. First, the linearized operator restricted to the infinite-dimensional kernel of the linearization at 0 , expressed with the explicit formula of an arbitrary approximate solution, gives an operator (see $\mathcal{M}_{0}$ below) we need to invert and for which we need to show the same properties as in [4]. Second, we need to control the small divisor problem which arises on the complement of this kernel. This proof follows the same path as in [4], which was largely inspired by the proof in [5] (problem with a fluid layer of finite depth), so we recall some essential steps and check precisely in the general case whether the proof made in [4] is still valid, modulo some adaptation and painful computations.

Acknowledgements The authors deeply acknowledge John Toland for many fruitful discussions.

\section{Linearized operator}

Let us define the perturbation $u$ by

$$
w=w_{\varepsilon}^{(N)}+\varepsilon^{N} u, \quad N \geq 4, \quad \mu=\varepsilon^{2} / 4
$$

and decompose as in [4] the linearized operator at such non zero point $w$

$$
\partial_{w} \mathcal{F}\left(w_{\varepsilon}^{(N)}+\varepsilon^{N} u, \varepsilon^{2} / 4\right)=\Lambda(u, \varepsilon)+\Gamma\left(\mathcal{F}\left(w, \varepsilon^{2} / 4\right), L_{w^{\prime}}(\cdot)\right)
$$

where $\Gamma$ cancels when $\mathcal{F}=0$, and satisfies suitable estimates (see [4]). Now the structure of the linear operator $\Lambda$ is detailed in [4], and this is the approximate linearized operator, we need to invert. Indeed, the linear equation for $\delta u(x, t)$

$$
\Lambda(u, \varepsilon) \delta u=f
$$

takes, after a number of transformations (see Theorem 7.5 of [4]), the form of a new equation for $\vartheta(\xi, \tau)$

$$
\mathcal{A}^{(0)} \vartheta=h
$$

where

$$
\begin{aligned}
\delta u & =L_{w^{\prime}}^{-1}\left\{\left(1+d^{\prime}\right)(\mathcal{P} \vartheta \circ \widehat{Q})\right\} \\
h & =\mathcal{P}^{-1}\left\{p_{1}\left(f \circ \widehat{Q}^{-1}\right)\right\}
\end{aligned}
$$

and

$$
\mathcal{A}^{(0)} \vartheta \equiv \partial_{\tau \tau} \vartheta-\left(1+\beta^{(0)}\right) \mathcal{H} \partial_{\xi} \vartheta-\kappa^{(0)} \vartheta-\mathcal{W}(\vartheta)
$$

The definitions of the coefficients $\beta^{(0)}=O\left(\varepsilon^{2}\right), \kappa^{(0)}=O\left(\varepsilon^{4}\right)$, the functions $d^{\prime}, p_{1}$, the change of coordinates $\widehat{Q}$ and the change of variables $\mathcal{P}$ are recalled below. The operator $\mathcal{W}$ is the sum of smoothing operators and quasi-one-dimensional operators, all of order $O(\varepsilon)$, and the above coefficients, functions and operators depend on $u$ in a "tame" way (see [4]), which is necessary for applying the Nash-Moser implicit function theorem. More precisely, we decompose (7) as follows

$$
\begin{aligned}
\mathcal{M}_{\varepsilon} \Theta+\mathcal{A}_{\varepsilon}^{(0)} \Psi & =\varepsilon^{-2} P_{0} h \\
\left(\Lambda_{\varepsilon}^{(0)}+\varepsilon \Lambda_{\varepsilon}^{(1)}\right) \Psi+\left(\mathcal{K}_{-1}+\varepsilon \mathcal{K}_{\varepsilon}\right) \Theta & =\varepsilon^{-1}\left(\mathbb{I}-P_{0}\right) \mathbb{P} h
\end{aligned}
$$


where $P_{0}$ and $\mathbb{P}$ are orthogonal projections in $L_{\text {如 }}^{2}$, resp. on ker $\mathcal{L}_{0}$, and on the set of functions of 0 average

$$
\vartheta=\Theta+\varepsilon \Psi, \quad \Theta=P_{0} \vartheta \in \operatorname{ker} \mathcal{L}_{0},
$$

and operators $\mathcal{M}_{\varepsilon}, \mathcal{A}_{\varepsilon}^{(0)}, \Lambda_{\varepsilon}^{(0)}, \Lambda_{\varepsilon}^{(1)}, \mathcal{K}_{-1}, \mathcal{K}_{\varepsilon}$ are defined by

$$
\begin{aligned}
\varepsilon^{2} \mathcal{M}_{\varepsilon} & =P_{0} \mathcal{A}^{(0)} P_{0} \\
\varepsilon \mathcal{A}_{\varepsilon}^{(0)} & =P_{0} \mathcal{A}^{(0)} \mathbb{P}\left(\mathbb{I}-P_{0}\right), \\
\Lambda_{\varepsilon}^{(0)}+\varepsilon \Lambda_{\varepsilon}^{(1)} & =\left(\mathbb{I}-P_{0}\right) \mathbb{P} \mathcal{A}^{(0)} \mathbb{P}\left(\mathbb{I}-P_{0}\right), \\
\varepsilon \mathcal{K}_{-1}+\varepsilon^{2} \mathcal{K}_{\varepsilon} & =\left(\mathbb{I}-P_{0}\right) \mathbb{P} \mathcal{A}^{(0)} P_{0} \\
\Lambda_{\varepsilon}^{(0)} \Psi=\partial_{\tau \tau} \Psi & -\left(1+\beta^{(0)}\right) \mathcal{H} \partial_{\xi} \Psi-\kappa^{(0)} \Psi .
\end{aligned}
$$

It is shown in [4] that the operator $\mathcal{W}$ is sufficiently smoothing in $\xi$ or in $\tau$, first for allowing to bound $\mathcal{M}_{\varepsilon}^{-1} \mathcal{A}_{\varepsilon}^{(0)}$ provided $\mathcal{M}_{0}^{-1}$ is good enough (as in [4], see Lemma 6 below), and second to be able to invert

$$
\Lambda_{\varepsilon}^{(0)}+\varepsilon \Lambda_{\varepsilon}^{(1)}-\left(\mathcal{K}_{-1}+\varepsilon \mathcal{K}_{\varepsilon}\right) \mathcal{M}_{\varepsilon}^{-1} \mathcal{A}_{\varepsilon}^{(0)}
$$

provided some diophantine conditions are realized by the coefficients $\beta^{(0)}, \kappa^{(0)}$ for insuring a suitable inverse for $\Lambda_{\varepsilon}^{(0)}$. The aim of all what follows is to give the principal parts of coefficients $\beta^{(0)}$ and $\kappa^{(0)}$, which appear to have the same form as in [4], and to compute $\mathcal{M}_{0}$ (and its inverse, see Lemma 6), and to show that the occurence of the new operator $\mathcal{K}_{-1}$, which is 0 in [4], does not perturb the invertibility of the operator

$$
\Lambda_{\varepsilon}^{(0)}-\mathcal{K}_{-1} \mathcal{M}_{0}^{-1} \mathcal{A}_{0}^{(0)},
$$

(see Lemma 7 and Theorem 8). The computation of $\mathcal{M}_{0}$ and the study of its invertibility and the invertibility of operator (13) are the main difficulties here.

\subsection{Definition of coefficients and changes of coordinates}

We first need, for any $w \in H_{\natural \natural}^{m, e e}$, to compute the functions $a \in H_{\natural \natural}^{m-1, o o}, b \in H_{\natural \natural}^{m-2, e e}$ occuring in lemma 5.1 of [4], defined by

$$
\begin{gathered}
a=\mathcal{H}\left(\frac{1}{D} L_{w^{\prime}} \dot{w}\right)+\frac{1}{D} \mathcal{H}\left(L_{w^{\prime}} \dot{w}\right) \\
b=D^{-1}\left\{a^{2} L_{w^{\prime}} w^{\prime \prime}-2 a L_{w^{\prime}} \dot{w}^{\prime}+L_{w^{\prime}} \ddot{w}+\mu\left(D-1-\mathcal{H} w^{\prime}\right)\right\}+\frac{L_{w^{\prime}} w^{\prime \prime}}{D^{3}}\left(\pi_{0} L_{w^{\prime}} \dot{w}\right)^{2} .
\end{gathered}
$$

Then we introduce the function $d(x, t) \in C_{\text {如 }}^{m-3, o e}$ defined by the linear PDE

$$
\partial_{t} d=a\left(1+\partial_{x} d\right),\left.\quad d\right|_{t=0}=0 .
$$

This defines the following change of coordinates

$$
\begin{aligned}
y & =x+d(x, t) \\
\tilde{u}(y, t) & =u(x, t), \quad \hat{v}(x, t)=v(y, t),
\end{aligned}
$$

and allows to introduce two important functions $q(y, t)$ and $p(y, t)$

$$
\begin{aligned}
& q=\left\{\left[\left(1+\varepsilon^{2} / 4\right)-\widetilde{b}\right]\left(\widetilde{1+d^{\prime}}\right)\right\} \\
& p=1-\partial_{y} \widetilde{d}=\left\{\left(1+d^{\prime}\right)^{-1}\right\} .
\end{aligned}
$$


Then we define the coefficient $\beta^{(0)}$ and two useful functions $d_{0}(y)$ and $e_{0}(y, t)$ by:

$$
\left(1+\partial_{t} e_{0}(y, t)\right)^{2}=\frac{q(y, t)\left(1+d_{0}^{\prime}(y)\right)}{1+\beta^{(0)}}
$$

where

$$
\frac{1}{2 \pi} \int_{-\pi}^{\pi}\{q(y, t)\}^{1 / 2} d t=\left(\frac{1+\beta^{(0)}}{1+d_{0}^{\prime}(y)}\right)^{1 / 2},
$$

and where we take the positive root in (19), and we use the fact that the average of the derivative of a periodic function is 0 . The changes of coordinates $Q$ and $\widehat{Q}$ are the following

$$
\begin{aligned}
& (\xi, \tau)=Q(y, t)=\left(y+d_{0}(y), t+e_{0}(y, t)\right) \\
& (\xi, \tau)=\widehat{Q}(x, t)=\left(x+d(x, t)+d_{0}(x+d(x, t)), t+e_{0}(x+d(x, t), t)\right)
\end{aligned}
$$

and the coefficient $\kappa^{(0)}$ and function $p_{1}(\xi, \tau)$ are defined by

$$
\begin{gathered}
\kappa^{(0)}=\frac{\left(1+\beta^{(0)}\right)^{2}}{16 \pi^{2}} \int_{-\pi}^{\pi} \int_{-\pi}^{\pi} \frac{\left(1+\dot{e}_{0}\right)}{\left(1+d_{0}^{\prime}\right)}\left\{\left(\frac{\ddot{e}_{0}}{q}\right)^{2}-\left(e_{0}^{\prime}\right)^{2}\right\} d t d y, \\
p_{1}=\left(\frac{p}{\left(1+\dot{e}_{0}\right)^{2}}\right) \circ Q^{-1} .
\end{gathered}
$$

We shall make precise later the near identity change of variables $\mathcal{P}$ (see (30)), with the computation of the main order of the operator $\mathcal{W}$.

\subsection{Calculation of coefficients in (9)}

We can show the following

Lemma 2 When $w_{\varepsilon}^{(N)}+\varepsilon^{N} u, \quad N \geq 4, \mu=\varepsilon^{2} / 4$ we obtain

$$
\begin{aligned}
a(x, t) & =2 \varepsilon \sum_{q \in I} \frac{\varepsilon_{q}}{q} \sin q^{2} x \sin q t+\varepsilon^{2} a^{(2)}(x, t)+O\left(\varepsilon^{3}\right) \\
b(x, t) & =-2 \varepsilon \sum_{q \in I} \varepsilon_{q} \cos q^{2} x \cos q t+\varepsilon^{2} b^{(2)}(x, t)+O\left(\varepsilon^{3}\right) \\
d(x, t) & =2 \varepsilon \sum_{q \in I} \frac{\varepsilon_{q}}{q^{2}} \sin q^{2} x(1-\cos q t)+\varepsilon^{2} d^{(2)}(x, t)+O\left(\varepsilon^{3}\right), \\
q(y, t) & =1+2 \varepsilon \sum_{q \in I} \varepsilon_{q} \cos q^{2} y+\varepsilon^{2} q^{(2)}(y, t)+O\left(\varepsilon^{3}\right), \\
p(y, t) & =1-2 \varepsilon \sum_{q \in I} \varepsilon_{q} \cos q^{2} y(1-\cos q t)+O\left(\varepsilon^{2}\right),
\end{aligned}
$$

with

$$
\begin{aligned}
a^{(2)}(x, t)= & -\sum_{\substack{r, s \in I \\
\varepsilon_{r}}} \varepsilon_{s} \frac{2 s}{r^{2}} \sin \left(r^{2}+s^{2}\right) x \cos r t \sin s t+ \\
& +\sum_{\substack{r, s \in I \\
r>s}} \varepsilon_{r} \varepsilon_{s} \frac{2 r}{s^{2}} \sin \left(r^{2}-s^{2}\right) x \sin r t \cos s t
\end{aligned}
$$




$$
\begin{aligned}
b^{(2)}(x, t)= & -\sum_{r \in I} 1+\sum_{r, s} \varepsilon_{r} \varepsilon_{s} \frac{2 s^{2}}{r^{2}} \cos \left(r^{2}+s^{2}\right) x \cos r t \cos s t+ \\
& +\sum_{r>s} 2 \varepsilon_{r} \varepsilon_{s} \cos \left(r^{2}-s^{2}\right) x\left\{\left(1-\frac{r^{2}}{s^{2}}\right) \cos r t \cos s t-\frac{r}{s} \sin r t \sin s t\right\}, \\
d^{(2)}(x, t)= & \sum_{r, s \in I} \varepsilon_{r} \varepsilon_{s} \frac{2}{r^{2}} \sin \left(r^{2}+s^{2}\right) x \cos r t(\cos s t-1)+ \\
& +\sum_{\substack{r, s \in I \\
r>s}} \varepsilon_{r} \varepsilon_{s} 2 \sin \left(r^{2}-s^{2}\right) x\left\{\frac{-s^{2}}{r^{2}\left(r^{2}-s^{2}\right)}-\frac{1}{r^{2}} \cos r t+\right. \\
& \left.+\frac{1}{s^{2}} \cos s t+\left(\frac{1}{r^{2}-s^{2}}-\frac{1}{s^{2}}\right) \cos r t \cos s t+\frac{s}{r\left(r^{2}-s^{2}\right)} \sin r t \sin s t\right\}, \\
q^{(2)}(y, t)= & \frac{1}{4}+\sum_{r \in I}(2-\cos 2 r t)-\sum_{r, s} 2 \frac{s^{2}}{r^{2}} \varepsilon_{r} \varepsilon_{s} \cos \left(r^{2}+s^{2}\right) y+ \\
& -\sum_{\substack{r, s \in I \\
r>s}} 2 \varepsilon_{r} \varepsilon_{s} \cos \left(r^{2}-s^{2}\right) y\left\{\cos r t \cos s t-\frac{r^{2}+s^{2}}{r s} \sin r t \sin s t-\frac{r^{2}}{s^{2}}\right\}
\end{aligned}
$$

Proof: The proof of this lemma is straightforward. Notice that for computing $d(x, t)$ we just need to identify powers of $\varepsilon$ in the PDE (16).

We can then show the following

Lemma 3 When $w_{\varepsilon}^{(N)}+\varepsilon^{N} u, N \geq 4, \mu=\varepsilon^{2} / 4$, we obtain

$$
\begin{gathered}
\beta^{(0)}=\frac{\varepsilon^{2}}{4}+O\left(\varepsilon^{3}\right), \quad \kappa^{(0)}=\frac{\varepsilon^{4}}{4}(\operatorname{card}(I)-1 / 2) \sum_{q \in I} q^{2}+O\left(\varepsilon^{5}\right) . \\
d_{0}(y)=-2 \varepsilon \sum_{q \in I} \frac{\varepsilon_{q}}{q^{2}} \sin q^{2} y+O\left(\varepsilon^{2}\right), \\
e_{0}(y, t)=\varepsilon^{2} \sum_{\substack{r, s \in I \\
r>s}} \frac{\varepsilon_{r} \varepsilon_{s}}{r s\left(r^{2}-s^{2}\right)} \cos \left(r^{2}-s^{2}\right) y\left\{s^{3} \sin r t \cos s t-r^{3} \cos r t \sin s t\right\}+ \\
-\varepsilon^{2} \sum_{r \in I} \frac{1}{4 r} \sin 2 r t+O\left(\varepsilon^{3}\right) .
\end{gathered}
$$

Proof: From the expression of $q(y, t)$ and (20), we obtain

$$
\begin{aligned}
\frac{1+d_{0}^{\prime}(y)}{1+\beta^{(0)}}= & 1-2 \varepsilon \sum_{q \in I} \varepsilon_{q} \cos q^{2} y-\frac{1}{4} \varepsilon^{2}+ \\
& +\varepsilon^{2} \sum_{\substack{r, s \in I \\
r^{2}}} 2\left(\frac{s^{2}}{r^{2}}+1\right) \varepsilon_{r} \varepsilon_{s} \cos \left(r^{2}+s^{2}\right) y+ \\
& +\varepsilon^{2} \sum_{\substack{r, s \in I \\
r>s}} 2 \varepsilon_{r} \varepsilon_{s}\left(2-\frac{r^{2}}{s^{2}}\right) \cos \left(r^{2}-s^{2}\right) y
\end{aligned}
$$


which leads to the results of the lemma for $\beta^{(0)}, d_{0}(y)$ and $e_{0}(y, t)$. Now from the formula for $\kappa^{(0)}$ we have

$$
\kappa^{(0)}=\frac{1}{16 \pi^{2}} \int_{-\pi}^{\pi} \int_{-\pi}^{\pi}\left\{\ddot{e}_{0}^{2}-e_{0}^{\prime 2}\right\} d y d t+O\left(\varepsilon^{5}\right)
$$

and after a straightforward calculation

$$
\kappa^{(0)}=\frac{\varepsilon^{4}}{4}\left(\sum_{r} \frac{r^{2}}{2}+\sum_{r>s}\left(r^{2}+s^{2}\right)\right)+O\left(\varepsilon^{5}\right)
$$

which leads to (27). We check that the formula fits with the case $I=\{1\}$ where $\kappa^{(0)}=$ $\varepsilon^{4} / 8+O\left(\varepsilon^{5}\right)($ see $[4])$.

\subsection{Calculation of the principal part of $\mathcal{W}$ in (9)}

As it is indicated in [4], the linear operator $\mathcal{W}$ is a sum of two parts as follows

$$
\mathcal{W}(\vartheta)=\left(\lambda_{0}+\lambda_{1} \mathcal{H}\right) \partial_{\tau}^{-2} \mathcal{P} \vartheta+\mathcal{V}(\vartheta)
$$

where we shall give later precisions on the part $\mathcal{V}(\vartheta)$. The near identity bounded operator $\mathcal{P}^{-1}$ is written as

$$
\mathcal{P}^{-1}=1+\alpha_{0}+\beta_{0} \mathcal{H}+\left(\alpha_{1}+\beta_{1} \mathcal{H}\right) \partial_{\tau}^{-1}+\left(\alpha_{2}+\beta_{2} \mathcal{H}\right) \partial_{\tau}^{-2}
$$

where $\partial_{\tau}^{-1}$ is defined by

$$
\partial_{\tau}^{-1} \cos n \tau=(1 / n) \sin n \tau, \quad \partial_{\tau}^{-1} \sin n \tau=-(1 / n) \cos n \tau, \partial_{\tau}^{-1} 1=0,
$$

and functions $\alpha_{j}, \beta_{j}, \lambda_{0}, \lambda_{1}$ are at most of order $\varepsilon$ (see Lemma M.3 of [4]). More precisely, let us show the following

Lemma 4 When $w=w_{\varepsilon}^{(N)}+\varepsilon^{N} u, \quad N \geq 4, \mu=\varepsilon^{2} / 4$, the coefficients $\lambda_{0}$ and $\lambda_{1}$ in the operator $\mathcal{W}\left(\right.$ see (29)) are $O\left(\varepsilon^{2}\right)$.

Remark: This means that the order $\varepsilon$ in $\mathcal{W}$ necessarily comes from the part $\mathcal{V}$. Moreover, the precisions we give below on the order of magnitude of coefficients $\alpha_{j}, \beta_{j}$ will be helpful for finding the principal part of $\mathcal{V}$.

Proof of the lemma: The computation of the above coefficients is based on the following 3 functions

$$
\begin{aligned}
\gamma & =\left\{\frac{\ddot{e}_{0}}{\left(1+\dot{e}_{0}\right)^{2}}\right\} \circ Q^{-1}, \quad \delta=-\left(1+\beta^{(0)}\right)\left(\frac{e_{0}^{\prime}}{1+d_{0}^{\prime}}\right) \circ Q^{-1}, \\
\alpha & =\left(\frac{-\partial_{y} q}{\left(1+\dot{e}_{0}\right)^{2}}\right) \circ Q^{-1},
\end{aligned}
$$

and it is clear from the form of $q$ and $e_{0}$ that $\alpha$ only depends on $\tau$ at order $\varepsilon^{2}$. Then considering formulas given in Appendix $\mathrm{M}$ of [4], giving $\alpha_{j}, \beta_{j}$, we obtain successively

$$
\alpha_{0}(\xi, \tau)=2 \varepsilon \sum_{q \in I} \varepsilon_{q}\left(\cos q^{2} \xi-1\right)+O\left(\varepsilon^{2}\right)
$$

and $\beta_{0}=O\left(\varepsilon^{2}\right), b_{1}, b_{2}=O\left(\varepsilon^{2}\right), \alpha_{1}, \beta_{1}=O\left(\varepsilon^{2}\right), b_{3}, b_{4}=O\left(\varepsilon^{2}\right), \alpha_{2}, \beta_{2}=O\left(\varepsilon^{2}\right), \lambda_{1}, \lambda_{2}=$ $O\left(\varepsilon^{2}\right)$. Hence the lemma is proved.

Now we are ready to compute the principal part, which is of order $\varepsilon$ in the operator $\mathcal{V}$ : we have the following 
Lemma 5 when $w=w_{\varepsilon}^{(N)}+\varepsilon^{N} u, N \geq 4, \mu=\varepsilon^{2} / 4$, the linear operator $\mathcal{V}$ satisfies

$\mathcal{V}(\vartheta)=2 \varepsilon \sum_{q \in I,} \sum_{n<q^{2}, p \in \mathbb{N}_{0}} \frac{\varepsilon_{q}\left(q^{2}-n\right)}{q^{2}}\{-n \cos q \tau \cos p \tau+p q \sin q \tau \sin p \tau\} \vartheta_{n}^{(p)} \cos \left(q^{2}-n\right) \xi+O\left(\varepsilon^{2}\right)$.

In particular, we have $P_{0} \mathcal{V} P_{0}=O\left(\varepsilon^{2}\right)$.

Remark: All the above formulae fit with what we found in [4] in the case when $I=\{1\}$, where we notice that $\mathcal{V}(\Theta)=O\left(\varepsilon^{2}\right)$ (instead of $O(\varepsilon)$ ), this order $O\left(\varepsilon^{2}\right)$ was very helpful in [4], since such a case implies $\mathcal{K}_{-1}=0$ (see (12) and (13)).

Proof: see Appendix 1.

\section{Inversion of the approximate linearized operator}

In this section we compute the operators $\mathcal{M}_{0}\left(\mathcal{M}_{\varepsilon}\right.$ is defined in $\left.(10)\right), \mathcal{A}_{0}^{(0)}$ and $\mathcal{K}_{-1}$ (defined in (12)). These operators are fundamental in the study of the inversion of the approximate linearized operator $\mathcal{A}^{(0)}$. Our aim is to prove the same type of estimates as in [4], despite of the occurence of the new operator $\mathcal{K}_{-1}$. Moreover we shall see that the form of $\mathcal{M}_{0}$ is more complicated than in the unimodal case, and that this leads to the need of the extra assumption $\mathrm{H}(\mathrm{I})$ of the subset $I \subset \mathbb{N}$ occuring in the asymptotic expansions of our standing waves.

Let us first observe that for $\Theta=\sum_{r \in \mathbb{N}} A_{r} \cos r^{2} \xi \cos r \tau \in \operatorname{ker} \mathcal{L}_{0}$ we already have from Lemma 5, two operators occuring in (13)

$$
\mathcal{K}_{-1} \Theta=2 \sum_{q \in I, r<q} \frac{r \varepsilon_{q}}{q^{2}} \partial_{\tau} \partial_{\xi}\left\{\cos q \tau \sin r \tau \sin \left(q^{2}-r^{2}\right) \xi\right\} A_{r}
$$

and

$$
\left\{\mathcal{A}_{0}^{(0)} \Psi\right\}_{q}=-\sum_{r \in I, r \geq q} \frac{\varepsilon_{r} q^{3}}{r^{2}}\left\{(r+q) \psi_{r^{2}-q^{2}}^{(r+q)}-(r-q) \psi_{r^{2}-q^{2}}^{(r-q)}\right\}
$$

hence it remains to compute $\mathcal{M}_{0}$. Indeed, we can show the following

Lemma 6 When $w=w_{\varepsilon}^{(N)}+\varepsilon^{N} u, N \geq 4, \mu=\varepsilon^{2} / 4$, we have for $\Theta=\sum_{p \in \mathbb{N}} A_{p} \cos p^{2} \xi \cos p \tau$

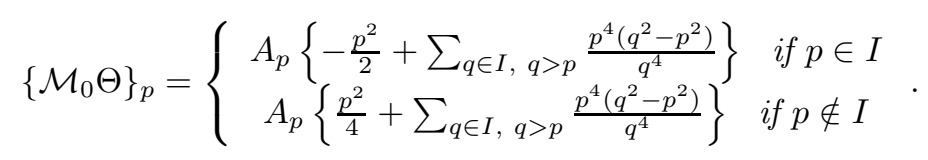

If Hypothesis $H(I)$ (see (4)) holds, operator $\mathcal{M}_{0}$ has a bounded inverse from $P_{0} H_{\text {घh }}^{\text {s,ee onto }}$ $P_{0} H_{\text {如 }}^{s+1, e e .}$

Remark: The above smoothing property of $\mathcal{M}_{0}^{-1}$ is precisely the one which is required in $[4]$.

Proof: For computing $\mathcal{M}_{0}$ we might compute the coefficient $\mathcal{W}^{(2)}$ of $\varepsilon^{2}$ in the operator $\mathcal{W}$. This is awful and we prefer to use a way which uses the calculations made in [3]. This lemma 6 is proved in Appendix 2.

Once $\mathcal{M}_{0}$ has a nice inverse as in [4], this leads to the need to invert the operator (13) (instead of $\Lambda_{\varepsilon}^{(0)}$ ). We observe that $\mathcal{A}_{0}^{(0)}$ and $\mathcal{K}_{-1}$ have finite dimensional matrices, since the subset of integers $I$ is finite, and since $\mathcal{M}_{0}^{-1}$ is diagonal the operator $\mathcal{K}_{-1} \mathcal{M}_{0}^{-1} \mathcal{A}_{0}^{(0)}$ has a finite dimensional matrix. More precisely, we have the following 
Lemma 7 When $w=w_{\varepsilon}^{(N)}+\varepsilon^{N} u, \quad N \geq 4, \mu=\varepsilon^{2} / 4$, we have

$$
-\left\{\mathcal{K}_{-1} \mathcal{M}_{0}^{-1} \mathcal{A}_{0}^{(0)} \Psi\right\}_{n}^{(l)}=0 \text { if }(n, l) \neq\left(q^{2}-p^{2}, q \pm p\right), \quad q \in I, 1 \leq p<q,
$$

and for $q \in I, 1 \leq p<q$

$$
\begin{aligned}
-\left\{\mathcal{K}_{-1} \mathcal{M}_{0}^{-1} \mathcal{A}_{0}^{(0)} \Psi\right\}_{p^{2}-q^{2}}^{(q-p)} & =-\sum_{r \in I, r \geq p} \frac{\varepsilon_{q} \varepsilon_{r} p^{2}\left(q^{2}-p^{2}\right)(q-p)}{q^{2} r^{2} m_{p}}\left\{(r+p) \Psi_{r^{2}-p^{2}}^{(r+p)}-(r-p) \Psi_{r^{2}-p^{2}}^{(r-p)}\right\} \\
-\left\{\mathcal{K}_{-1} \mathcal{M}_{0}^{-1} \mathcal{A}_{0}^{(0)} \Psi\right\}_{p^{2}-q^{2}}^{(q+p)} & =\sum_{r \in I, r \geq p} \frac{\varepsilon_{q} \varepsilon_{r} p^{2}\left(q^{2}-p^{2}\right)(q+p)}{q^{2} r^{2} m_{p}}\left\{(r+p) \Psi_{r^{2}-p^{2}}^{(r+p)}-(r-p) \Psi_{r^{2}-p^{2}}^{(r-p)}\right\}
\end{aligned}
$$

where

$$
m_{p}=\left\{\begin{array}{cc}
-\frac{1}{2}+\sum_{q \in I, q>p} \frac{p^{2}\left(q^{2}-p^{2}\right)}{q^{4}} & \text { if } p \in I, \\
\frac{1}{4}+\sum_{q \in I, q>p} \frac{p^{2}\left(q^{2}-p^{2}\right)}{q^{4}} & \text { if } p \notin I .
\end{array}\right.
$$

Proof: The proof of this lemma follows immediately from

$$
\left\{\mathcal{M}_{0}^{-1} \mathcal{A}_{0}^{(0)} \Psi\right\}_{p}=-\sum_{r \in I, r \geq p} \frac{\varepsilon_{r} p}{r^{2} m_{p}}\left\{(r+p) \psi_{r^{2}-p^{2}}^{(r+p)}-(r-p) \psi_{r^{2}-p^{2}}^{(r-p)}\right\}
$$

and from the form of $\mathcal{K}_{-1}$, noticing that $\left(r^{2}-p^{2}, r \pm p\right)=\left(r^{\prime 2}-p^{\prime 2}, r^{\prime} \pm p^{\prime}\right)$ leads to $r=r^{\prime}$, $p=p^{\prime}$.

Now we can show the following

Theorem 8 Assume that $\beta^{(0)}$ and $\kappa^{(0)}$ satisfy the diophantine condition

$$
\left|-q^{2}+\left(1+\beta^{(0)}\right) p-\kappa^{(0)}\right| \geq c / q^{2}, \text { for } p \neq q^{2},
$$

then, provided that assumption $H(I)$ holds $\left(m_{p} \neq 0\right.$ for $\left.p \in I\right)$ the linear operator

$$
\Lambda_{\varepsilon}^{(0)}-\mathcal{K}_{-1} \mathcal{M}_{0}^{-1} \mathcal{A}_{0}^{(0)}
$$

has an inverse which satisfies the estimate

$$
\left\|\left\{\Lambda_{\varepsilon}^{(0)}-\mathcal{K}_{-1} \mathcal{M}_{0}^{-1} \mathcal{A}_{0}^{(0)}\right\}^{-1} \Psi\right\|_{H^{s}} \leq c(s)\|\Psi\|_{H^{s+2}},
$$

i.e. as for the inverse of $\Lambda_{\varepsilon}^{(0)}$ (see [4]).

Proof: For $l^{2}+n>2(\max \{I\})^{2},\left\{-\mathcal{K}_{-1} \mathcal{M}_{0}^{-1} \mathcal{A}_{0}^{(0)} \Psi\right\}_{n}^{(l)}=0$, hence for large $n$ and $l$ the operator reduces to $\Lambda_{\varepsilon}^{(0)}$. Hence, to prove the above theorem, it is sufficient to prove that for components $\Psi_{n}^{(l)}$ with $l^{2}+n \leq 2(\max \{I\})^{2}$, the linear operator $\Lambda_{\varepsilon}^{(0)}-\mathcal{K}_{-1} \mathcal{M}_{0}^{-1} \mathcal{A}_{0}^{(0)}$ is invertible. Indeed, it is sufficient to consider components of the form $\Psi_{q^{2}-p^{2}}^{(q \pm p)}$ where $q \in I$, and $p \leq q$, since these components are uncoupled from the rest where the operator is diagonal. We then need to invert the following linear operator acting on components $\Psi_{q^{2}-p^{2}}^{(q \pm p)}$

$$
\begin{aligned}
& \Psi_{q^{2}-p^{2}}^{(q-p)}-\sum_{r \in I, r \geq p} \frac{\varepsilon_{q} \varepsilon_{r} p\left(q^{2}-p^{2}\right)}{2 q^{2} r^{2} m_{p}}\left\{(r+p) \Psi_{r^{2}-p^{2}}^{(r+p)}-(r-p) \Psi_{r^{2}-p^{2}}^{(r-p)}\right\} \\
& \Psi_{q^{2}-p^{2}}^{(q+p)}-\sum_{r \in I, r \geq p} \frac{\varepsilon_{q} \varepsilon_{r} p\left(q^{2}-p^{2}\right)}{2 q^{2} r^{2} m_{p}}\left\{(r+p) \Psi_{r^{2}-p^{2}}^{(r+p)}-(r-p) \Psi_{r^{2}-p^{2}}^{(r-p)}\right\}
\end{aligned}
$$

which has a simple structure with respect to $D_{q^{2}-p^{2}}=\Psi_{q^{2}-p^{2}}^{(q+p)}-\Psi_{q^{2}-p^{2}}^{(q-p)}$ and $S_{q^{2}-p^{2}}=$ $\Psi_{q^{2}-p^{2}}^{(q+p)}+\Psi_{q^{2}-p^{2}}^{(q-p)}$. Since, the operator reduces to the identity for $D_{q^{2}-p^{2}}$, we just need to 
invert with respect to $S_{q^{2}-p^{2}}$ the linear operator with components (for fixed $p \geq 1$ and all $q>p$, since we already have $D_{0}=S_{0}$ )

$$
m_{p} \frac{\varepsilon_{q}}{q^{2}} S_{q^{2}-p^{2}}-\frac{p^{2}\left(q^{2}-p^{2}\right)}{q^{4}} \sum_{r \in I, r>p} \frac{\varepsilon_{r}}{r^{2}} S_{r^{2}-p^{2}} .
$$

Taking the sum of all lines we obtain

$$
\left(m_{p}-\sum_{q \in I, q>p} \frac{p^{2}\left(q^{2}-p^{2}\right)}{q^{4}}\right) \sum_{r \in I, r>p} \frac{\varepsilon_{r}}{r^{2}} S_{r^{2}-p^{2}}
$$

and since the factor in front is $-1 / 2$ or $1 / 4$ we can solve with respect to the sum $\sum_{r \in I, r>p} \frac{\varepsilon_{r}}{r^{2}} S_{r^{2}-p^{2}}$, hence solve with respect to all unknowns, and theorem 8 is proved. It results from Lemma 6 and Theorem 8 that the method of [4] applies exactly.

\subsection{Kernel of $\mathcal{A}^{(0)}$}

To complete the proof of theorem 1 , we just need to specify ker $\mathcal{A}^{(0)}$. Indeed in [4] we use the precise structure of $\mathcal{W}(1)$ at order $\varepsilon^{2}$, which we have not in this general case. In fact the kernel also satisfies $\vartheta^{(0)}=1+O(\varepsilon)$ in the general case, which is precisely what we need to show.

Since constants lie in $\operatorname{ker} \partial_{w} \mathcal{F}(w, \mu)$, this implies that the function $\vartheta^{(1)}$ defined by (see(8))

$$
\vartheta^{(1)}=\mathcal{P}^{-1}\left\{\left(p\left(\widetilde{1+\mathcal{H} w^{\prime}}\right)\right) \circ Q^{-1}\right\}
$$

satisfies

$$
\mathcal{A}^{(0)} \vartheta^{(1)}=h_{1}=O\left(\varepsilon^{N+1}\right)
$$

due to the fact that $\mathcal{A}^{(0)}$ is a reformulation of operator $\Lambda$, and that the "error" term $\Gamma=$ $O\left(\varepsilon^{N+1}\right)$ in (5). Let us proceed as in [4] and define the kernel $\vartheta^{(0)}$ in fixing its component on constants equal to $1: \vartheta^{(0)}=1+\varpi_{0}, \quad \mathbb{P} \varpi_{0}=0$. Let us also decompose $\vartheta^{(1)}=\gamma_{1} 1+\varpi_{1}$, $\mathbb{P} \varpi_{1}=0$, then $\gamma_{1}=1+O(\varepsilon)$ and $\varpi_{1}=O(\varepsilon)$ by construction, and since $\vartheta^{(1)}$ and $\vartheta^{(0)}$ are as smooth as we wish, we can now use the inverse of $\mathcal{A}^{(0)}$ restricted to the space of 0 -average functions, which is $O\left(\varepsilon^{-2}\right)$ as soon as diophantine conditions are satisfied by $\beta^{(0)}$ and $\kappa^{(0)}$ :

$$
\mathcal{A}^{(0)}\left(\varpi_{1}-\gamma_{1} \varpi_{0}\right)=h_{1}
$$

hence, since $N \geq 4$

$$
\varpi_{0}=\gamma_{1}^{-1}\left\{\varpi_{1}-\left(\mathbb{P} \mathcal{A}^{(0)} \mathbb{P}\right)^{-1} \mathbb{P} h_{1}\right\}=O(\varepsilon) .
$$

All required properties which are used in [4] are satisfied, the proof of theorem 1 is then completed.

\section{Appendix 1}

We want now to give the principal part of the operator $\mathcal{V}$ in theorem 7.5 of [4]. For this calculation, we first need to compute the main order of the operator $\mathcal{G}$ in theorem 6.9 of [4], where

$$
\mathcal{G}(\varphi)=-\partial_{y}\left\{\mathcal{S}(q \varphi)+\mathcal{S}_{q} \varphi+\left(\mathcal{H} \mathcal{S}_{a}\left(\widehat{\partial_{t} \varphi / p}\right)\right)^{\sim}\right\}
$$

where by definition, for any smooth enough function $f$

$$
\mathcal{S} f(y, t)=(\mathcal{H} \hat{f})^{\sim}(y, t)-(\mathcal{H} f)(y, t), \quad \mathcal{S}_{a} f=\mathcal{H}(a f)-a \mathcal{H} f .
$$


Since $p=1+O(\varepsilon), a=O(\varepsilon)$, and the change of coordinates $(x, t) \mapsto(y, t)$ is $\varepsilon$ - close to the identity, we obtain thanks to $(22)$

$$
\left(\mathcal{H} \mathcal{S}_{a}\left(\widehat{\partial_{t} \varphi / p}\right)\right)^{\sim}=2 \varepsilon \sum_{q \in I} \frac{\varepsilon_{q}}{q} \sin q t \mathcal{H} \mathcal{S}_{\sin q^{2} y} \partial_{t} \varphi(y, t)+O\left(\varepsilon^{2}\right) .
$$

We also have from (I.2) in Appendix I of [4]

$$
\mathcal{S}(q \varphi)=\mathcal{S}_{\widetilde{d}}(q \varphi)^{\prime}+O\left(\varepsilon^{2}\right),
$$

it then results (using (24) and (25)), that

$$
\begin{aligned}
\mathcal{G}(\varphi)= & -2 \varepsilon \partial_{y} \sum_{q \in I} \frac{\varepsilon_{q}}{q^{2}}\left\{\partial_{y}\left(\mathcal{S}_{\sin q^{2} y} \varphi\right)-\cos q t \mathcal{S}_{\sin q^{2} y} \varphi^{\prime}\right\}+ \\
& -2 \varepsilon \partial_{y} \sum_{q \in I} \frac{\varepsilon_{q}}{q} \sin q t \mathcal{H} \mathcal{S}_{\sin q^{2} y} \partial_{t} \varphi+O\left(\varepsilon^{2}\right) .
\end{aligned}
$$

Now comes the computation of the main order of the operator $\mathcal{G}_{0}$ in lemma 7.2 of [4]. Since $\beta^{(0)}$ and $\delta$ are $O\left(\varepsilon^{2}\right)$ and using again (I.2) in [4]

$$
\mathcal{S}_{(0)} \theta=(\mathcal{H}(\theta \circ Q)) \circ Q^{-1}-\mathcal{H} \theta=\mathcal{S}_{\widetilde{d_{0}}} \theta^{\prime}+O\left(\varepsilon^{2}\right)=O(\varepsilon)
$$

where $\widetilde{d}_{0}(\xi)$ is $d_{0}(y)$ expressed with $\xi=y+d_{0}(y)$, and since the mapping $Q$ is $\varepsilon$ - close to the identity (see (21)), we have

$$
\mathcal{G}_{0}(\theta)=-\partial_{\xi} \mathcal{S}_{\widetilde{d_{0}}} \theta^{\prime}+\mathcal{G}(\theta)+O\left(\varepsilon^{2}\right)
$$

Now, from the formula giving $\mathcal{V}$ in Appendix $\mathrm{M}$ of [4], we have

$$
\mathcal{V}(\vartheta)=-\mathcal{G}_{0}(\vartheta)-\partial_{\xi} \mathcal{S}_{\alpha_{0}} \vartheta+O\left(\varepsilon^{2}\right)
$$

and thanks to the forms of $d_{0}$ (see Lemma 3 ) and $\alpha_{0}$ (see (31)) at order $\varepsilon$, we obtain

$$
\mathcal{V}(\vartheta)=2 \varepsilon \sum_{q \in I} \frac{\varepsilon_{q}}{q^{2}} \partial_{\xi}\left\{-\cos q \tau \mathcal{S}_{\sin q^{2} \xi} \theta^{\prime}+q \sin q \tau \mathcal{H} \mathcal{S}_{\sin q^{2} \xi} \partial_{\tau} \theta\right\}+O\left(\varepsilon^{2}\right) .
$$

Now using the identities

$$
\begin{aligned}
& \mathcal{H S}_{\sin q^{2} \xi} \cos n \xi=-\sin \left(q^{2}-n\right) \xi, \quad-n \mathcal{S}_{\sin q^{2} \xi} \sin n \xi=n \sin \left(q^{2}-n\right) \xi \quad \text { for } n<q^{2} \\
& \mathcal{H S}_{\sin q^{2} \xi} \cos n \xi=0, \quad-n \mathcal{S}_{\sin q^{2} \xi} \sin n \xi=0 \quad \text { for } n \geq q^{2}
\end{aligned}
$$

we obtain the result of Lemma 5 .

\section{$5 \quad$ Appendix 2}

In this appendix, we prove Lemma 6 . To reach the operator $\mathcal{M}_{0}$ let us use the calculations made in [3]. In Lemma 1 of [3] we show that the map $w \mapsto v=F(w)$ defined by

$$
\begin{aligned}
v & =F(w)=w+B\left(w, w^{\prime}\right) \\
B\left(w_{1}, w_{2}\right) & =\mathcal{H}\left(w_{1} w_{2}\right)-w_{1} \mathcal{H} w_{2}-2 w_{2} \mathcal{H} w_{1}
\end{aligned}
$$

transforms equation (2) into a new one $\mathcal{E}(v, \mu)=0$ for $v$, where there is no longer any quadratic term in $v$. Moreover

$$
\mathcal{F}(w, \mu)=\widetilde{\Gamma}_{w} \mathcal{E}(v, \mu)
$$


where $\widetilde{\Gamma}_{w}$ is the inverse linear map of $f \mapsto f+\partial_{x} B(w, f)$. Hence defining $v_{\varepsilon}^{(N)}=F\left(w_{\varepsilon}^{(N)}\right)$, we have $\mathcal{E}\left(v_{\varepsilon}^{(N)}, \frac{\varepsilon^{2}}{4}\right)=O\left(\varepsilon^{N+1}\right)$ and from Lemma 1 of [3], the above definitions and from the definition of operator $\Lambda$ in (5) $(N \geq 4)$, we obtain

$$
\Lambda(u, \varepsilon)=\mathcal{L}_{0}+\varepsilon\left\{\widetilde{\Gamma}^{(1)} \mathcal{L}_{0}+\mathcal{L}_{0} f^{(1)}\right\}+\varepsilon^{2}\left\{\widetilde{\Gamma}^{(2)} \mathcal{L}_{0}+\widetilde{\Gamma}^{(1)} \mathcal{L}_{0} f^{(1)}+\mathcal{L}_{0} f^{(2)}+\mathcal{B}_{2}\right\}+O\left(\varepsilon^{3}\right),
$$

with

$$
\begin{aligned}
& \widetilde{\Gamma}_{w_{\varepsilon}^{(N)}}=\mathbb{I}+\varepsilon \widetilde{\Gamma}^{(1)}+\varepsilon^{2} \widetilde{\Gamma}^{(2)}+O\left(\varepsilon^{3}\right), \quad \widetilde{\Gamma}^{(1)}=-\partial_{x} B\left(w^{(1)}, \cdot\right), \\
& \partial_{w} F\left(w_{\varepsilon}^{(N)}\right)=\mathbb{I}+\varepsilon f^{(1)}+\varepsilon^{2} f^{(2)}+O\left(\varepsilon^{3}\right), \quad f^{(1)}=B\left(\cdot, w^{(1) \prime}\right)+B\left(w^{(1)}, \partial_{x} \cdot\right) \\
& \partial_{v} \mathcal{E}\left(v_{\varepsilon}^{(N)}, \frac{\varepsilon^{2}}{4}\right)=\mathcal{L}_{0}+\varepsilon^{2} \mathcal{B}_{2}+O\left(\varepsilon^{3}\right),
\end{aligned}
$$

and $\mathcal{B}_{2}$ satisfies (see the proof of Theorem 4 of [3]) for $\Theta=\sum_{r \in \mathbb{N}} A_{r} \cos r^{2} \xi \cos r \tau \in \operatorname{ker} \mathcal{L}_{0}$

$$
\left\{P_{0} \mathcal{B}_{2} \Theta\right\}_{p}=\left\{\begin{array}{cc}
-\frac{p^{2}}{2} A_{p}, & \text { for } p \in I, \\
\frac{p^{2}}{4} A_{p}, & \text { for } p \notin I .
\end{array}\right.
$$

Coming back to the relationship between operators $\Lambda$ and $\mathcal{A}^{(0)}$ (see (6), (7) and (8)), we can check that for $\Theta=P_{0} \vartheta$, that

$$
\begin{aligned}
\mathcal{A}^{(0)} \Theta= & \varepsilon \mathcal{L}_{0}\left(\mathcal{Q}^{(1)}+f^{(1)}\right) \Theta+\varepsilon^{2}\left\{\left(\widetilde{\Gamma}^{(1)}+\widetilde{\mathcal{Q}}^{(1)}+p_{1}^{(1)}-\mathcal{P}^{(1)}\right) \mathcal{L}_{0}\left(f^{(1)}+\mathcal{Q}^{(1)}\right)+\mathcal{B}_{2}+\right. \\
& \left.+\mathcal{L}_{0}\left(f^{(2)}+f^{(1)} \mathcal{Q}^{(1)}+\mathcal{Q}^{(2)}\right)\right\} \Theta+O\left(\varepsilon^{3}\right),
\end{aligned}
$$

where

$$
\begin{aligned}
p_{1}^{(1)} & =-2 \sum_{q \in I} \varepsilon_{q} \cos q^{2} \xi(1-\cos q \tau), \quad \mathcal{P}^{(1)}=2 \sum_{q \in I} \varepsilon_{q}\left(1-\cos q^{2} \xi\right), \\
Q^{(1)} \vartheta & =\sum_{q \in I} \varepsilon_{q}\left\{\left(2-\cos q^{2} x \cos q t\right) \vartheta-\left(\mathcal{H} \vartheta+\frac{2 \partial_{x} \vartheta}{q^{2}}\right) \sin q^{2} x \cos q t\right\} \\
\widetilde{\mathcal{Q}}^{(1)} g & =2 \sum_{q \in I} \frac{\varepsilon_{q} \partial_{\xi} g}{q^{2}} \sin q^{2} \xi \cos q \tau .
\end{aligned}
$$

Since $P_{0} \mathcal{L}_{0}=0$, we finally have

$$
\mathcal{M}_{0} \Theta=P_{0}\left\{\left(\widetilde{\Gamma}^{(1)}+\widetilde{\mathcal{Q}}^{(1)}+p_{1}^{(1)}-\mathcal{P}^{(1)}\right) \mathcal{L}_{0}\left(f^{(1)}+\mathcal{Q}^{(1)}\right)+\mathcal{B}_{2}\right\} \Theta .
$$

Let us first consider $\mathcal{L}_{0}\left(f^{(1)}+\mathcal{Q}^{(1)}\right) \Theta$ : we have

$$
f^{(1)} \Theta=B\left(\Theta, w^{(1) \prime}\right)+B\left(w^{(1)}, \Theta^{\prime}\right)
$$

hence

$$
\left(f^{(1)}+\mathcal{Q}^{(1)}\right) \Theta=\partial_{\xi} \mathcal{S}_{w^{(1)}} \Theta+2 \Theta \sum_{q \in I} \varepsilon_{q}
$$

and since $\mathcal{L}_{0} \Theta=0$, and

$$
\mathcal{S}_{\cos q^{2} \xi} \cos n \xi=\left\{\begin{array}{c}
-\sin \left(q^{2}-n\right) \xi, \text { for } n<q^{2} \\
=0 \text { for } n \geq q^{2}
\end{array}\right.
$$

then we obtain

$$
\begin{aligned}
& \mathcal{L}_{0}\left(f^{(1)}+\mathcal{Q}^{(1)}\right) \Theta \\
= & \sum_{q \in I, 1 \leq r<q} \frac{\varepsilon_{q} r\left(q^{2}-r^{2}\right)}{q^{2}} A_{r} \cos \left(q^{2}-r^{2}\right) \xi\{(q+r) \cos (q+r) \tau-(q-r) \cos (q-r) \tau\} .
\end{aligned}
$$


Now, we can check that

$$
\left\{P_{0}\left(\widetilde{\Gamma}^{(1)}+\widetilde{\mathcal{Q}}^{(1)}+p_{1}^{(1)}-\mathcal{P}^{(1)}\right) \phi\right\}_{p}=-2 \phi_{p^{2}}^{(p)} \sum_{s \in I} \varepsilon_{s}+\sum_{s \in I, 1 \leq p<s} \frac{\varepsilon_{s} p^{2}}{2 s^{2}}\left\{\phi_{s^{2}-p^{2}}^{(s-p)}+\phi_{s^{2}-p^{2}}^{(s+p)}\right\},
$$

and observing that $\phi_{p^{2}}^{(p)}=0$, and collecting the above results, we obtain

$$
\left\{P_{0}\left(\widetilde{\Gamma}^{(1)}+\widetilde{\mathcal{Q}}^{(1)}+p_{1}^{(1)}-\mathcal{P}^{(1)}\right) \mathcal{L}_{0}\left(f^{(1)}+\mathcal{Q}^{(1)}\right) \Theta\right\}_{p}=A_{p} \sum_{q \in I, 1 \leq p<q} \frac{p^{4}\left(q^{2}-p^{2}\right)}{q^{4}} .
$$

Now thanks to (32), we have completely $\mathcal{M}_{0}$ as given in Lemma 6 . We notice that the operator $\mathcal{M}_{0}$ is diagonal, and that the factor of $A_{p}$ is $>0$ for $p \notin I$, in particular for large $p$. We then need to assume that assumption $\mathrm{H}(\mathrm{I})$ holds for $p \in I$. This assumption $\mathrm{H}(\mathrm{I})$ insures that for $p \geq 1$, one has

$$
\left|\left\{\mathcal{M}_{0} \Theta\right\}_{p}\right| \geq c p^{2}\left|A_{p}\right|
$$

i.e. the inverse $\mathcal{M}_{0}^{-1}$ has the same smoothing properties as in [4], and lemma 6 is proved.

\section{References}

[1] A.I.Dyachenko, E.A.Kuznetsov, M.D.Spector, V.E.Zakharov. Analytic description of the free surface dynamics of an ideal fluid. Physics Lett. A 221 (1996), 73-79.

[2] G.Iooss. On the standing wave problem in deep water. J.Math. Fluid Mech. 4 (2002), 155-185.

[3] G.Iooss, P.Plotnikov. Multimodal standing gravity waves: a completely resonant system. J.Math. Fluid Mech. (same volume)

[4] G.Iooss, P.I.Plotnikov, J.F.Toland. Standing waves on an infinitely deep perfect fluid under gravity. Preprint March 2004.

[5] P.I.Plotnikov, J.F.Toland. Nash-Moser theory for standing water waves. Arch. Rat. Mech. Anal. 159, (2001) 1-83. 